\title{
Access to Knowledge of Spatial Structure at Novel Points of Observation
}

\author{
John J. Rieser \\ Vanderbilt University
}

\begin{abstract}
Adults were asked to judge the self-to-object directions in a room from novel points of observation that differed from their actual point at times only by a rotation and at other times only by a translation. The results show for the rotation trials that the errors and latencies when a novel point was imagined were worse than the baseline responses from their actual points of observation, and the latencies varied as a function of the magnitude of the to-be-imagined rotation. For the translation trials, on the other hand, the errors and latencies when a novel point was imagined were comparable to the baseline responses from their actual point and did not vary significantly across the different imagined station points. The evidence indicates that subjects know the objectto-object relations directly, without going through the origin of a coordinate system. In addition, similarities in processing during imagination on the one hand, and perception and action on the other are discussed.
\end{abstract}

The spatial structure of a place consists of the distances and directions relating its objects, features, and events. Observers often produce spatially coordinated action while on the move and plan actions before reaching the station points from which they intend to launch them. This is the case when one plans a route before embarking on a trip. And it is implied whenever one launches an act while on the move, because the motor plan to control the act must be set before the launching point is reached. Because of this, observers need access to knowledge of the spatial structure of places from novel station points. The present experiments are about observers' abilities to imagine the spatial structure available at novel points of observation and about some of the conditions that facilitate access to such knowledge. In each experiment observers stood at one point of observation and viewed the locations of target objects scattered around them in a room. Then they were asked to close their eyes, imagine they occupied a novel point of observation, and aim a pointer at the targets relative to the novel point.

Geometrically, movements to new points of observation consist of combinations of simple rotation and translation

Parts of the manuscript were written while the author was on leave at the Laboratoire de Psychology de L'Enfant, and the hospitality of Henriette Bloch and the Laboratory is gratefully acknowledged.

The research was supported in part by Grant HD 15051 from the National Institutes of Child Health and Human Development to Vanderbilt University.

Experiment 2 was conducted with the help of David Guth and David Weatherford, who contributed to all phases of it.

In addition, I thank Steven Kramer and Jean-Claude Lepecq for help in understanding the findings, Elizabeth Rider for help in testing subjects, and Michael Young, Timothy McNamara, and Laura Novick for help in improving the manuscript.

Correspondence concerning this article should be addressed to John Rieser, Department of Psychology and Human Development, Box 512, Peabody, Vanderbilt University, Nashville, Tennessee 37203. movements. Preliminary to conducting the present experiments, we practiced imagining the structure at novel points of observation that differed from our actual points by simple rotations versus simple translations. Subjectively, it seemed easier to imagine the structure after translations than rotations and after some rotations than others. Theoretically, the relative ease of accessing knowledge of spatial structure at novel station points after rotations versus translations depends on the organization of the underlying spatial knowledge and processes used to access it. Thus, facts about the relative ease of access can be used to constrain models of the underlying processes and knowledge representations. In addition, they provide an opportunity to explore similarities between imagination on the one hand and perception and action on the other.

\section{Organization of Spatial Knowledge}

James J. Gibson (1979) argued that observers perceive the invariant structure of a place, not the structure of their own perspectives. By perspective structure he meant the "personcentered" distances and directions relating an observer occupying a particular station point (i.e., location and facing direction) to features of the surrounding environment, a structure that continually changes during locomotion. By invariant structure he meant the "environment-centered," object-toobject distances and directions that are constant across temporary variations in an observer's point of observation (Sedgwick, 1983). Gibson noted that observers flexibly launch actions from numerous station points, and he theorized that their actions are guided by perception of the invariant structure of a place. However, flexible access per se does not demonstrate the use of invariant structure because knowledge of the perspective structure at any station point is a logically sufficient basis from which to compute the structure at any other point, given knowledge of appropriate computations. 


\section{Geometry of Simple Movements}

Consider a computational model drawn from analytic geometry of an observer locomoting through a fixed environment and keeping up to date on the changing self-to-object distances and directions. Within the model, an observer's movements to new station points would correspond to rotations and translations of the origin and axes of a coordinate system through a field of objects whose locations are constant but whose coordinates change relative to the moving coordinate system. The number of computational steps needed to keep up-to-date on the changing coordinates depends on the coordinate system used and on the type of movement. Consider two examples: the computations needed for a twodimensional space when given a polar coordinate system and when given a rectangular coordinate system. Given a polar coordinate system, it takes fewer steps to compute target locations relative to the new, moved coordinate system after simple rotations of the axes than after simple translations. Given rectangular coordinate systems, the reverse is true, and it takes fewer steps to compute target locations relative to the new system after simple translations of the old system (Coxeter, 1968).

\section{Overview}

The purpose of the present three experiments was to investigate adult observers' access to knowledge of the spatial structure of a place from novel station points that differed from the actual ones by rotations or by translations. The introspections of laboratory members were that it was easier to judge the structure available at novel station points that differed by translations than by rotations. The purpose of Experiment 1 was to assess the generality of those introspections to larger groups of naive observers. The difficulty of imagining rotations seemed to increase as a function of increasing amounts of rotation in station point. This would be true if performance were mediated by processes analogous to visual scanning and physical rotation in order to judge target directions from novel station points. The purpose of Experiment 2 was to assess the effect of magnitude of rotation from the actual station point on the latency and accuracy of judgments. Finally, the purpose of Experiment 3 was to compare the latency and accuracy of judgments directly across conditions where the novel station points differed from the actual ones only by a rotation versus only by a translation.

There is a logical difficulty in comparing performance across two qualitatively different scales of movement like rotations and translations in order to conclude that one is more difficult than the other. Namely, how many degrees of rotation does one elect to compare with which distances of translation? What is needed is a method to ensure that similarly representative samples of each type of movement are selected for comparison. The approach in the present study was to assess performance after rotations and after translations for the same space. That is, subjects were asked to judge the directions toward the same sets of target objects and to judge the directions from novel points of observation that were identified by the same locations in the space. So for rotations, subjects were asked to judge directions relative to the novel facing direction (keeping the same location) identified by a set of objects in the surroundings. For the translations, subjects were asked to judge directions relative to the novel locations (keeping the same facing direction) identified by the same set of objects.

\section{Experiment 1}

This experiment was a study of subjective reports of the relative difficulty of imagining rotations and translations. The procedures were uncomplicated, and interested readers can follow the methods to obtain informal demonstrations of the phenomenon on themselves and others.

\section{Method}

Twenty college and graduate students participated. The tests were conducted in eight different offices and living rooms. Subjects sat roughly centered in the room and were asked to study and remember the locations of five objects scattered around them. Different objects were used in the different rooms, but each was selected so it was 1-3 $\mathrm{m}$ from the subject and so the different objects were spaced at uneven intervals all around the subjects. After 2 or $3 \mathrm{~min}$ of study, subjects closed their eyes and were asked to point at each target object as it was named in a random order by the experimenter. Subjects were free to study the target objects again until they could point easily to within about $20^{\circ}$ according to the experimenter's judgment.

After this, subjects were asked to imagine occupying a novel point of observation and, imagining this, to point at the targets as they were named in a random order. In the rotation condition subjects were asked to "Point at the (target name) as if you are facing the (object name)." In the translation condition, subjects were asked to "Point at the (target name) as if you are standing at the (object name)." These instructions were demonstrated to the subjects, who then completed a block of 10 test trials for each condition. The objects identifying the facing direction and target object were randomly varied within each condition. Half of the subjects were tested first in the block of rotation condition trials, and the others underwent the translation trials first. After completion of both blocks, subjects were asked to say whether one block was more difficult than the other and, if so, to say which was more difficult and to rate the difference as slight, moderate, or great.

\section{Results and Discussion}

All 20 subjects reported that the rotation trials were more difficult than the translation trials. All rated the difference as moderate or great. In addition, all subjects reported that some of the rotation trials seemed more difficult than the others. Eleven subjects suggested that the trials involving longer degrees of imagined rotation were more difficult than the shorter rotations, and the others were uncertain about which were more difficult.

These different subjective experiences were universal across the subjects and consistent across the uncontrolled variations in test room and object location. Experiment 2 is about rotations only. It was designed to assess the effects on latency and accuracy of responding of the magnitude of rotation. 


\section{Experiment 2}

In this experiment subjects were asked to study an array of objects from one point of observation, close their eyes, and then aim a pointer at the target from a novel point, one with a novel facing direction. In order to assess the degree to which nonvisual locomotor information about rotations facilitates access to this knowledge, subjects were asked to judge these directions after physically rotating to the new point of observation or after imagining the rotation. In order to assess the effects of magnitude of rotation on performance, the actual and novel station points differed by $0^{\circ}$ through $320^{\circ}$ of rotation.

\section{Effects of Magnitude of Rotation}

The expected effects of magnitude of rotation on performance depend on the underlying model of performance. Consider the implications of a process model emphasizing the computations of analytic geometry and another emphasizing analogies to perception and action. For the computational model, one needs knowledge of the coordinates of the target objects and the facing object (no matter what coordinate system is used) and then needs to compute the new angle, using knowledge of trigonometric functions and of procedures to solve algebraic equations. Assuming that computations with small and large numbers involve the same time, the magnitude of the rotation would not influence processing speed or accuracy because the same computational steps would be needed for every value of rotation.

Shepard and Cooper (1982) summarized work pointing at similarities in perceiving and imagining objects. Similarities may occur in the perception and imagination of the environment as well. Suppose, for example, that the processes to access knowledge of one's surroundings when imagining a rotation in point of observation are analogous to physical exploratory acts such as visually scanning the surroundings in order to locate the facing object, physically rotating body position to face the new heading, and visually scanning again to locate the target object from the new station point. It would take longer to scan for points farther from straight ahead than closer to it, and this might lead to slower performance. In addition, it would take longer to rotate physically to points that were longer rotations than shorter ones; this would also lead to slower performance.

Amount of rotation is correlated with corresponding changes in two other variables, namely, amount of change in the correct response angle and reversals in the target's position around the observer's left-right axis. The change in response angle toward any object after a rotation equals the magnitude of the rotation itself and is opposite in (left-right) direction. The changes in a target's left-right position relative to an observer's body axes are more complicated. Consider the change in position of the field of possible locations relative to the left-right axis after $40^{\circ}$ and $80^{\circ}$ rotations for the experimental space used in the present experiment, which is depicted in Figure 1. After a $40^{\circ}$ rotation the locations in the segment of space subtended by the $40^{\circ}$ arc of the actual rotation are left-right reversed as are the locations subtended by the diametrically opposed $40^{\circ}$ degree arc, thereby reversing the locations of $80^{\circ}$ or $22.2 \%$ of the surrounding space. Similarly, an $80^{\circ}$ rotation reverses the locations of $160^{\circ}$ or $44.4 \%$ of space, and a $180^{\circ}$ rotation reverses the locations of $100 \%$ of space (see Figure 1).

There is evidence that left-right reversals pose significant psychological boundaries on processing in the context of numerous tasks. One example is the deficient discrimination performance of figures differing by a reflection around the left-right axis compared with reflections around the other axes (Appelle, 1972; Olson \& Bialystok, 1983). Another example is stimulus-response compatibility (Brebner, Shephard, \& Cairney, 1972), in which observers respond more rapidly

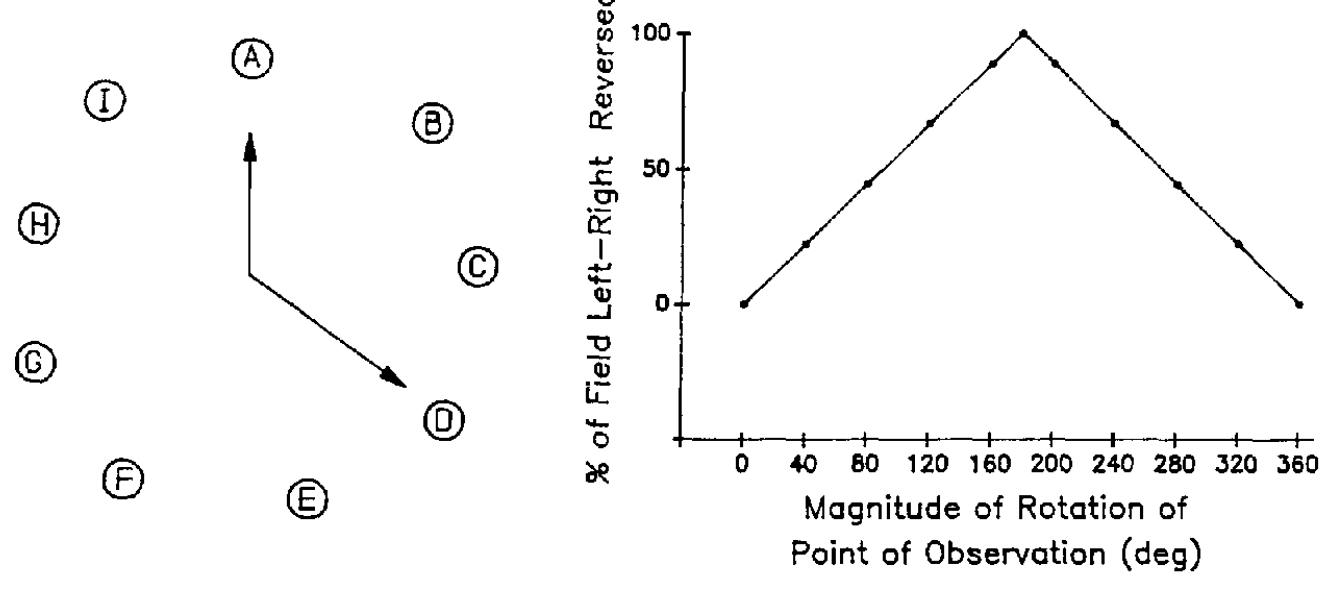

Figure 1. An array of targets like that used in Experiment 2 is depicted in the left hand side of the figure. (The circled letters mark the object locations, which were used to identify the novel station point and the target object. The arrows mark two possible station points, which were always centered within the array but faced in different directions. In the right-hand side of the figure is depicted a graph of the percentage of the locations in space that undergo a left-right reversal for each of the novel station points varying from $0^{\circ}$ to $360^{\circ}$ of rotation relative to the actual point.) 
to the left-right position of targets, with motor responses launched from the same half-field than the opposite half-field. If similar constraints operate on spatial orientation, then it would be more difficult to access knowledge after rotations that caused a reversal in left-right position than after rotations that did not cause a reversal, and this difference in difficulty would remain even for rotations that were the same in magnitude and direction. According to this, subjects would perform poorly after an $80^{\circ}$ rotation on targets located in the left-right reversed segments and would perform well after the same rotation on targets located out of the reversed segment.

Thus, a curvilinear effect of magnitude of rotation on performance could be due to the amounts of rotation per se, in which case processes analogous to visual scanning and/or physical rotation would be implicated. Alternatively, a curvilinear pattern could be due to corresponding changes in the percentages of reversal trials, in which case processes needed to reverse the left-right direction of the underlying code or of the response would be implicated.

\section{Access After Imagining Versus Locomoting to New Points of Observation}

Observers seem to have relatively easy and accurate access to knowledge of the spatial structure of a place after locomoting to new points of observation within the place, even when the place itself is occluded from view. Attneave and Farrar (1977) noted this when they asked adults to view targets in a room, turn away from the targets, and then judge their locations. Hardwick, McIntyre, and Pick (1976) found this when they asked children and adults to aim a sighting tube at targets after moving to new points of observation.

The relatively good access found in these experiments might have been mediated by information associated with the physical movement to the new point of observation per se or by partially overlapping views of some of the same features of the surrounding environment while standing at the initial point and while responding to it from the novel point. Research by Rieser, Guth, and Hill (1986) showed that the efference and/or proprioception from the locomotor movement per se facilitates access. Their subjects were asked to study an array of six target objects in a large room from one point of observation and then to judge the directions toward the targets from a novel point. After the study phase in the locomotion condition, subjects were blindfolded, guided to a new station point (one with a new location and a new facing direction), and asked to respond by aiming a pointer at each of the targets. In the imagination condition, subjects were blindfolded, guided to a new station point, guided back to the study position, asked to imagine being at the new station, and asked to respond. Although all subjects judged the directions with better than chance levels of accuracy, they responded more accurately and rapidly in the locomotion condition than in the imagination condition. Because subjects were blindfolded and outfitted with a sound system during the movements and tests, the resuits show that the efference and proprioception associated with the locomotion itself facilitated their access to knowledge of the structure at the novel points of observation.
Because of these findings, access to knowledge of spatial structure after locomoting without vision to new points seems to be mediated perceptually, whereas access, when subjects are asked to imagine new points, seems to be mediated by different, more effortful processes. In these previous studies the new points of observation differed by both a rotation and translation from the original point. It is not known whether the locomotion facilitates access more or less for rotations alone that vary in magnitude.

\section{Method}

Subjects and target array. Six college and graduate students participated as subjects. All were unfamiliar with the present research problems. On each trial, subjects were asked to aim a swivel mounted pointer in the direction of a target object from a novel station. The pointer was mounted above a $360^{\circ}$ protractor marked off in singledegree intervals and attached to a timer so that the clock stopped when subjects pushed the pointer. The target array, depicted in Figure 1 , consisted of nine common objects with one-syllable names equally spaced around a $2.5-\mathrm{m}$ diameter circle at $40^{\circ}$ intervals. It was located in a $6 \times 6-\mathrm{m}$ cluttered room that was cleared in the middle to display the targets. The purpose and procedures of the study were explained to subjects before they entered the test room. Included in this was a detailed explanation that sometimes they would be guided to a new point of observation and then asked to aim the pointer at one of the targets, and at other times they would be asked to imagine being at a new point and then aim the pointer. The instructions were repeated and demonstrated until the subjects understood them.

Procedures and design. Subjects stood centered in the array and were asked to study the targets in order to learn their names and locations. During the study phase, subjects stood always facing the same single target but were free to turn their heads to survey the entire field. The study phase lasted 5-10 min and continued until subjects could close their eyes and aim the pointer at each target within about $1 \mathrm{~s}$ of latency and within about $10^{\circ}$ of error. Subjects were instructed to respond as rapidly as they could and maintain accuracy.

During each of the 90 repeated tests, subjects were instructed to close their eyes and "Point at the (target name) as if facing the (object name)." There was a 1-s pause after the target was named, and subjects were asked to remember the target position then. Feedback was not provided. The response latency and direction were recorded, subject and pointer were returned to the constant starting position, and then subjects were asked to open their eyes and look around at the targets before the next trial. Subjects were asked to respond as rapidly as they could and still aim their responses accurately. Target locations and facing directions were always identified by the nine objects circling the subjects. Half the subjects participated first in the locomotion condition and half in the imagination condition. In both conditions the experimenter started the timer as he identified the facing direction, and subjects stopped the timer automatically by pushing the pointer after responding.

During the locomotion trials, subjects were guided to the new facing direction. To do this, the experimenter grasped them firmly from behind by the shoulders and rotated them. The rotation was timed with the instructions so that it ended at the same time as the new facing direction was identified verbally. During the imagination condition, subjects did not physically move and were simply asked to imagine facing the new direction and respond.

One independent variable in the design is movement mode--that is, whether subjects locomoted to the new point of observation or merely imagined it. The other independent variable is the magnitude 
of rotation, that is, the test position's rotation compared with the study position. The positions marked by the nine targets were all used, and the magnitudes of rotation (all expressed in a clockwise direction) were $0^{\circ}, 40^{\circ}, 80^{\circ}, 120^{\circ}, 160^{\circ}, 200^{\circ}, 240^{\circ}, 280^{\circ}$, and $320^{\circ}$. If computed in the shortest directions, the corresponding rotations were $0^{\circ}, 40^{\circ}, 80^{\circ}, 120^{\circ}, 160^{\circ}$, in the clockwise direction, and $160^{\circ}, 120^{\circ}$, $80^{\circ}$, and $40^{\circ}$ in the counterclockwise direction.

It is important to note that the magnitude of the correct response angle varied across the trials. Observers are more accurate aiming pointers at targets located in front of them than behind them (Howard \& Templeton, 1966), and it is reasonable to assume that it would take longer to turn a pointer through the longer distances needed to aim it backwards toward targets behind them than through the shorter distances to aim it forwards. Thus, it is important that the angles of the correct responses not confound the independent variables in the design. To control this, the same trials were used for the locomotor and imagination modes. In addition, the same five response angles were used for each value of rotation, namely $0^{\circ}, 40^{\circ}, 80^{\circ}, 120^{\circ}$, and $160^{\circ}$, about half to the left and half to the right. This results in a total of 90 trials per subject ( 2 modes of movement $\times 9$ rotations $\times 5$ response angles). The dependent variables were the median response errors in degrees for each individual's five repeated trials in each cell and the median response latencies.

\section{Results}

The mean of each individual's median latencies and errors appear in Figure 2. They were positively correlated, $r=.58$, indicating that on the more difficult trials the subjects tended to respond more slowly and less accurately and that they did not trade speed for accuracy. The latency and error scores were submitted to separate Movement Mode $\times$ Rotation analyses of variance with repeated measures on both factors. For the latencies, there were statistically significant main effects of mode, $F(1,5)=21.10, p=.006$, and of rotation, $F(8,40)=10.0, p<.001$. The Mode $\times$ Rotation interaction was also significant, $F(8,40)=10.7, p<.001$. The interaction depicted in Figure 2 was caused by the presence of a strong curvilinear trend of the latencies as a function of the magnitude of rotation in the imagination condition and a flat, linear trend for the locomotion condition. For the errors, there was a significant main effect of mode, $F(1,5)=6.8, p=.047$, and none of the other effects approached statistical significance.

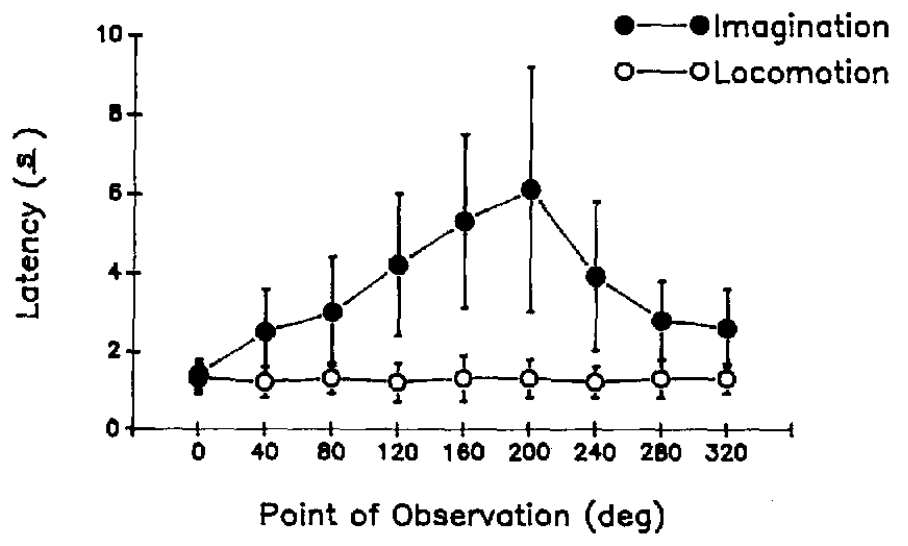

For the locomotion trials, performance was quite good, comparable to baseline performance during the trials with no rotation to a new station point. The average latency in the no-change trials was $1.2 \mathrm{~s}$, and the average latency across the change trials was the same and varied from 1.1 to $1.3 \mathrm{~s}$ across the different magnitudes of rotation. The average error in the no-change trials was $12^{\circ}$, and the average error across the change trials was the same and varied from $8^{\circ}$ to $16^{\circ}$ across the different magnitudes of rotation. Thus, the subjects responded as rapidly and accurately after locomoting to the new station point as from their actual station point. There is no evidence here to indicate that processing after the locomotion was needed.

For the imagination condition, baseline performance during the trials with no change in rotation was better than performance in the trials with imagined rotations to novel station points. The average latency for the no-change trials was $1.4 \mathrm{~s}$, and the average latencies for the eight different magnitudes of imagined rotation ranged from 2.8 through 6.2 $\mathrm{s}$; each was significantly different from the no-change latency by Tukey's test. The average $10^{\circ}$ error for the no-change trials was better than the errors for imagined rotation trials, which ranged from $13^{\circ}$ to $24^{\circ}$ for the different magnitudes of imagined rotation. All but the errors for the $40^{\circ}$ rotations significantly differed by Tukey's test.

\section{Discussion}

Subjects performed significantly more accurately and rapidly in the locomotion condition than the imagination condition, showing that the nonvisual information from locomotor rotations facilitated access to knowledge of spatial structure at the novel points. In the locomotion condition, responding was similar in the trials where no rotation was needed and in the other trials where the needed amounts of rotation ranged from $40^{\circ}$ through $340^{\circ}$. The processing needed to compensate for the rotation in point of observation seems to have been completed during the physical movement even before the novel station point was named.

During the imagination condition, on the other hand, subjects responded more rapidly and generally more accu-

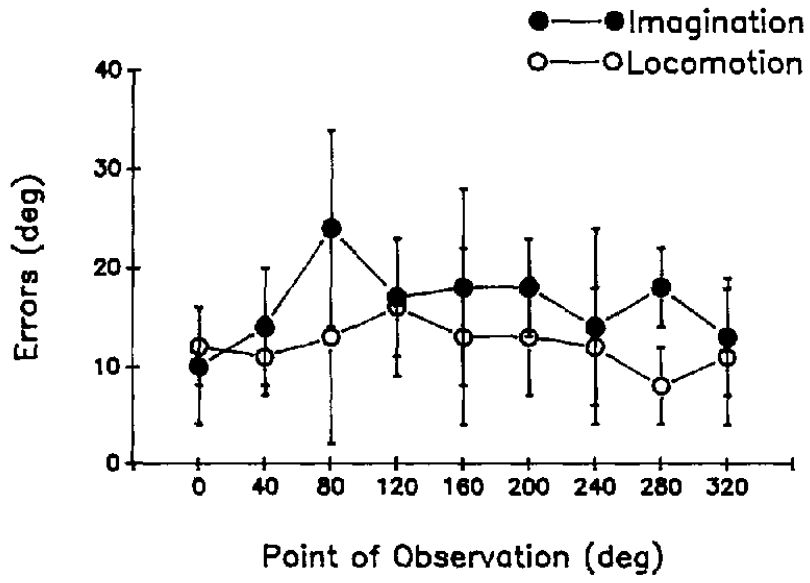

Figure 2. The mean latencies and errors of performance in Experiment 2, plotted as a function of the imagined versus locomotor mode of movement and of the magnitude of rotation. (Vertical lines = standard deviations.) 
rately in the condition with no rotation than in any of the others. This indicates that processing was needed to compensate for the rotation. The curvilinear effect of amount of rotation on the latencies shows that the amount of processing time was related to the amount of rotation relating to actual and imagined point of observation. Because the response requirements were the same in the locomotion condition as in the imagination condition, the curvilinear function in the imagination condition must have been caused by processing needed to determine the correct response, not with motor response requirements.

The proportion of trials resulting in a reversal of the target location around the left-right axis was also a curvilinear function of magnitude of rotation. To investigate the degree to which the curvilinearity of the latencies was associated with the magnitude of rotation per se, the frequency of left-right reversal trials, or with both, each test trial was cross classified on three dimensions: whether it involved a reversal of target position around the left-right axis, its degree of rotation in point of observation, and the angle of the correct response. Four pairs of matched trials, one a reversal and the other a nonreversal trial, had the same degree of rotation and same angle of correct response. Performance in the reversal trials (which averaged 3.2-s latency and $25^{\circ}$ error) did not significantly differ by $t$ test from performance in the nonreversal trials (which averaged 3.6-s latency and $32^{\circ}$ error). Thus, the effect of rotation is due to the magnitude of the change in facing direction, not to differences in proportions of trials reversing target position around the left-right axis.

The curvilinear trend for the latencies is consistent with the idea that the underlying processes are analogous to physical processes, requiring more time for longer distances than shorter distances. Four of the 6 individual subjects fit this pattern, showing monotonic patterns of latency for values of rotation ascending to $180^{\circ}$ and monotonically decreasing for values ascending on to $360^{\circ}$. The other two showed a similar pattern, but with one point out of order.

Hintzman, O'Dell, and Arndt (1981) conducted a long series of experiments that was in many ways similar to the present experiment. They obtained a similar curvilinear pattern in latencies after subjects studied a map of the test place, but not when they studied the place itself; however, a curvilinear trend after subjects studied the place was found here. In addition to procedural differences, the studies differed in one way that may account for the different pattern of findings.

In Hintzman et al., the targets were located at $45^{\circ}$ intervals starting at straight ahead, whereas in the present experiment $40^{\circ}$ intervals were used. Hintzman et al. pointed out that the use of the $45^{\circ}$ intervals would make available strategies that are not available given $40^{\circ}$ steps, strategies that do not involve mental rotation. For example, it might be relatively easy to respond to $180^{\circ}$ facing directions if subjects follow the strategy of merely reversing the actual target direction. In addition, $90^{\circ}$ and $270^{\circ}$ facing directions might be relatively easy for targets located at $0^{\circ}, 90^{\circ}, 180^{\circ}$, and $270^{\circ}$. The reason for this is that the response would line up with the observer's body axes, those directly to the front, back, left and right and, being cardinal directions, these responses and directions might be particularly easy to process. In the present experiment, $40^{\circ}$ steps were used to reduce subjects' opportunities to use alternative strategies such as these. However, $45^{\circ}$ steps were used in the next experiment in order to investigate the possible effects of step size.

\section{Experiment 3}

Experiment 3 was modeled after Experiment 2 in order to compare performance during imagined translations in point of observation versus imagined rotations. Preliminary data showed subjects performed quite well in the imagination mode with translations, achieving baseline levels of latency and accuracy, and therefore the locomotor versus imagination mode of movement to novel station points was not varied. The experiment was conducted to accomplish four goals. The first was to assess whether processing time was needed to respond during translation trials or whether subjects could access knowledge of the structure relatively directly. Increases in latency and/or error compared with baseline responding would indicate the need for additional processing of their knowledge of spatial structure of the target place. The second goal was to replicate the findings for processing time obtained for rotation trials in Experiment 2 and to evaluate the consequences of using $45^{\circ}$ steps.

The third goal was to compare performance after imagined translations versus imagined rotations in station point. There is no logically a priori way to equate amounts of rotation and translation in order to compare fairly selected samples of each type of movement. For comparison in the present experiment, the same target objects were used to identify the novel station points for the translation and the rotation trials. The fourth goal of the present experiment was to provide an additional test of the possible effects of whether the target position was reversed around the left-right axis.

\section{Method}

The methods were closely modeled after those in Experiment 2. The 6 subjects, who were college and graduate students, stood centered in an eight-item array of target objects, with one target straight ahead and the others equally spaced at $45^{\circ}$ intervals. After a study phase to the same criterion as in Experiment 2, subjects were asked on each trial to close their eyes and during translation trials to "Point at the (target name) as if standing at the (object name)." During rotation trials the instruction was to "Point at the (target name) as if facing the (object name)" the same as in Experiment 2. The instruction was explained for the translation condition and for the rotation condition before subjects entered the laboratory room, and subjects were pilot tested on their comprehension of it outside as well. All eight of the target positions served to identify the locations of novel points of observation from which subjects were asked to point at each of the other targets. In addition, subjects were asked to point at each of the targets from their actual point of observation. For the rotations, the station point identified by the target located at $0^{\circ}$ of rotation compared with their actual position resulted in trials where there was no change in point of observation. For the translations, however, the $0^{\circ}$ station point did involve a translation. To obtain a sample of performance during trials with no change in point of observation, subjects underwent 8 additional trials where they were instructed to "point at the (target name) as if standing at your true location." The total of 64 trials were randomly interspersed. Latencies and errors 
were recorded as in Experiment 2. Each subject participated in a set of translation trials and a set of rotation trials, half participating first on the set of rotations and the next day participating on the set of translations, and the other half participating first on the set of translations. Each session lasted about $1.5 \mathrm{hr}$ in duration.

\section{Results and Discussion}

Scores were assigned consisting of each subject's median latency and error in each condition across the seven trials with a new station point. The mean latencies and errors appear in Figure 3. They were positively correlated, $r(15)=$ .61 , indicating that the subjects responded more slowly and less accurately on the more difficult trials and did not trade speed of responding for accuracy. The latency and error scores were submitted to separate Movement Type $\times$ Station Point analyses of variance with repeated measures on both factors. For the latencies there were statistically significant main effects of movement type, $F(1,5)=51.5, p=.001$, and of station point, $F(7,35)=7.9, p<.001$. The Movement Type $\times$ Station Point interaction was also significant, $F(7,35)=$ $8.0, p<.001$. The interaction depicted in Figure 3 was caused by the presence of a strong curvilinear trend of the latencies as a function of station point for the rotation trials and a flat, linear trend for the translation trials.

For the translation trials, performance was quite good, comparable to baseline performance during the trials with no change in point of observation. The average latency in the no-change trials was $1.4 \mathrm{~s}$, the same as the average computed over all of the change trials, and the average error in the nochange trials was $16^{\circ}$, the same as the overall average error. Judging from Figure 3, there was no apparent effect of station point-that is, of the angle of the to-be-imagined novel points of observation from the actual point-on responding.

For the rotation trials, performance in the no-change trials with $0^{\circ}$ rotation was better than performance in the trials with $45^{\circ}$ to $180^{\circ}$ of rotation. The average latency for the $0^{\circ}$ nochange trials was $1.3 \mathrm{~s}$, whereas the latencies for the other blocks ranged from 1.8 to $3.2 \mathrm{~s}$, each of which significantly differed from the no-change trials by Tukey's post hoc tests. The average error for the $0^{\circ}$ no-change trials was $12^{\circ}$, whereas the errors for the other blocks ranged from $18^{\circ}$ to $37^{\circ}$. By Tukey's test, the errors for the $90^{\circ}$ and $225^{\circ}$ rotations did not significantly differ from the errors in the no-change condition, and the errors for the other amounts of rotation did significantly differ. Responding at the $180^{\circ}$ rotations was significantly faster at the $p<.01$ level of post hoc tests than at the $135^{\circ}$ and $225^{\circ}$ rotations. This is consistent with use of a rule simply to reverse the direction of the actual target location during some of the trials.

For the errors, the main effect of movement type approached significance, $F(1,5)=4.9, p=.076$, and the Movement Type $\times$ station point interaction approached significance as well, $F(7,35)=1.9, p=.10$. Only two of the translation test trials caused a reversal in target position around the left-right axis. Each of the reversal trials was matched to the single nonreversal trial in the set that had the same response angle and station. For the rotation trials, four of the reversals could be paired with nonreversal trials with the same novel facing direction and same correct response angle. The reversal and nonreversal trials did not significantly differ by Tukey's test.

\section{General Discussion}

Overall, the results show that performance during the trials that were translations was comparable to baseline performance where there was no change in station point. Thus, within the limits set by the sensitivity of these procedures, there is no evidence to indicate that any processing of knowledge of the spatial structure was needed beyond merely accessing it. For the rotation trials, on the other hand, performance was significantly worse than baseline performance, and the magnitude of the increase in latency was proportional to the magnitude of rotation. The analyses show, in addition, that these effects are not caused by associated differences in proportions of trials where target position was reversed around the left-right axis. Discussed below are the possible causes of the significant effects of the geometry of the imagined movement, the modality of movement in station point, and the magnitude of rotation in station point.
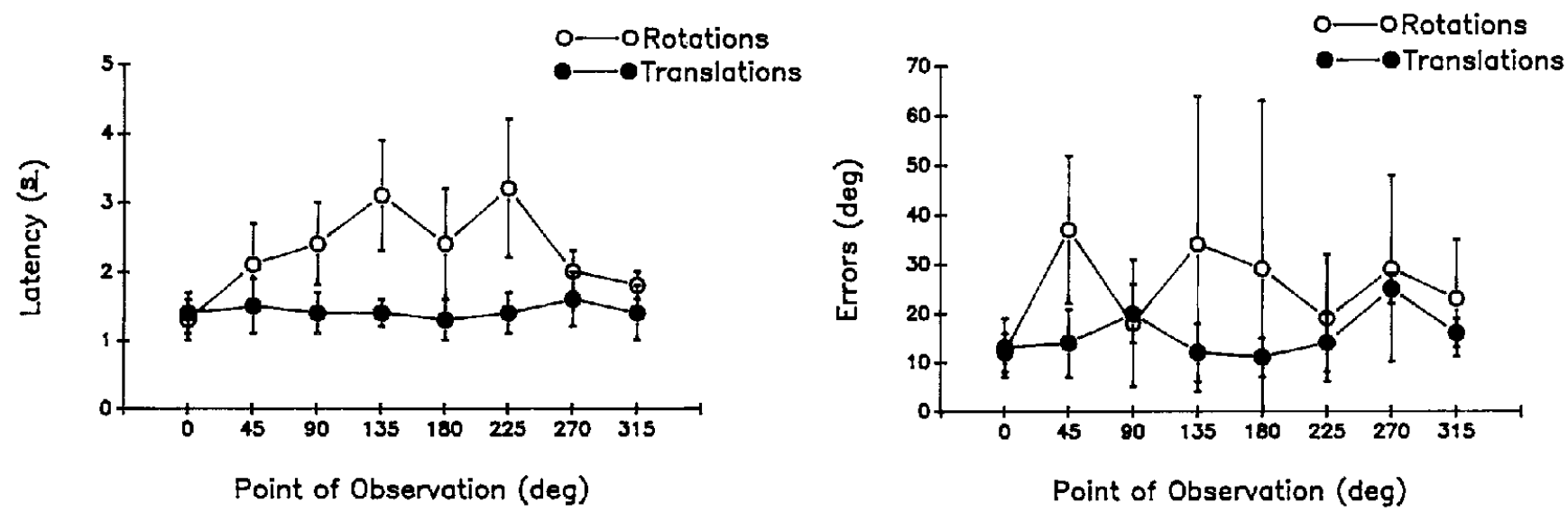

Figure 3. The mean latencies and errors of performance in Experiment 3, plotted as a function of rotation versus translation type of movement and the novel station point's location. (Vertical lines $=$ standard deviations.) 


\section{Geometry of Imagined Movements in Station Point}

The evidence shows that access to the novel directions for the translation trials was relatively direct, whereas for the rotation trials additional processing was needed. This implies that the knowledge accessed consisted of the object-to-object relations, not the self-to-object relations. During the translation trials, subjects needed to aim the pointer in the direction of the line connecting the target object and the novel station point relative to their own facing direction, which was constant. If subjects mentally represented the self-to-object relations of the situation and had direct access to their knowledge of them, then they would know the direction of each target relative to their own point of observation, but not relative to the other targets. In this case, one might suppose that processing time would be needed to compute the object-to-object relations. If, on the other hand, subjects mentally represented the object-to-object relations in the situation, then no processing time would be needed to compute them.

The converse is the case for performance on the rotation trials, for which performance was significantly slower and less accurate for the trials requiring an imagined rotation in station point compared with baseline performance. This indicates that subjects could not directly access knowledge of this relation and that they needed to engage in mental processing in order to determine it. Consider the implications for responding if subjects operated on their knowledge of the selfto-object versus object-to-object relations. During the rotation trials, subjects needed to aim the pointer at a target as if they faced one of the other targets. If subjects mentally represented the self-to-object relations, responding would be a matter of accessing the actual self-to-target direction, the self-to-station direction, and adding the two with appropriate signs. On the other hand, if subjects had direct access to the object-to-object relations (and not the self-to-object relations), then access would be even more problematic, requiring additional mental processes to determine the correct response.

\section{Locomotor Versus Imagined Mode of Movement to New Station Points}

The modality of movement to the new station points was varied for the rotation movements. The results were that subjects performed with baseline levels of latency and accuracy for the locomotor trials where they were actually guided to the new station point. This was not the case for the imagined rotation trials, however, for which latencies and errors both significantly differed during the trials with novel station points relative to the baseline trials from their actual points. For the imagination trials, the implication is that subjects needed additional processing time to determine the location of the novel station point relative to the subject's actual station.

For the locomotion trials, on the other hand, there is no evidence that processing in addition to that associated with the locomotor movement itself was needed. We believe that this reflects the nature of the perception of locomotion. Under the present conditions, observers rotated while their eyes were closed, and they were outfitted with a sound system, prohib- iting access to environmental information directly specifying their changing positions relative to objects fixed in the surroundings. Lee (1978) argued that perceptual systems could have been designed to operate by recording the temporal sequence of limb movements needed to implement locomotion, but that they were not designed in this way. Instead, he argued that much behavior is spatially coordinated with the surrounding environment, and it would therefore be adaptive for locomotion to be perceived in terms of the observer's changing position relative to it. The present findings are consistent with Lee's idea that locomotion is perceived in terms of the observer's position relative to the surroundings. This ability may be mediated by perceptual learning whereby observers notice the invariant relation between the optical flow fields and proprioceptive feedback generated during locomotion when moving without vision (Rieser, 1987; Rieser et al., 1986).

\section{Magnitude of Imagined Rotation in Station Point}

During the imagined rotation trials, latencies were a curvilinear function of the magnitude of rotation of the novel station point relative to the actual station. This was the case overall across Experiments 2 and 3. It was the case for 9 of the 12 individual subjects involved as well, and the other 3 subjects showed similar patterns. Consider the implications for whether the processes mediating performance are better thought of as computations or as analogous to perception and action.

Analytic geometry provides a useful computational model of the task and test situation. Changes in an observer's point of observation can be conveniently described as rotations and translations of the coordinate system. The calculations from analytic geometry by which one would compute the effects of movements of the coordinate system on the coordinates of stable objects provide explicit candidates for the psychological processes underlying performance. Furthermore, assuming that test performance is mediated by spatial knowledge that is organized like a rectangular coordinate system, an analytic geometry model of the calculational processes fits with the finding of easier performance after translations in point of observation than after rotations. However, contrary to the results of the experiments if the underlying processes were like these computations, then, depending on the algorithm used, one might suppose that the magnitude of rotation would not influence processing time.

If, on the other hand, the processes are analogous to perception and action, the response latencies should be a curvilinear function of rotation. Suppose subjects needed to scan their knowledge of the surroundings to locate the novel point of observation via processes analogous to visual scanning. If this is the case, then the time needed to scan in order to locate the novel station point would be proportional to the point's distance of rotation from the actual station and thus would be consistent with the results showing this relation.

However, the idea that scan time is the cause of the curvilinear function of the latencies for the rotation trials is not consistent with the translation data. The same targets were used to identify the novel station points in the translation and 
rotation trials. Because of this, one would expect the scan times to vary across the different station points in the same way during the translation trials as during the rotation trials. The results for the translation trials were that station point was not related to response latency or error. Overall, the results indicate that processes were not analogous to visual scanning but may have been analogous to physical rotation in station point.

The placement of the novel facing directions in $45^{\circ}$ steps in Experiment 3 influenced performance compared with the $40^{\circ}$ steps used in Experiment 2. The average 3.6-s latency obtained in the imagined rotation condition of Experiment 2 is slower than the 2.3-s latency obtained in the same conditions of Experiment 3. In addition, the average latency at the $180^{\circ}$ rotation was significantly faster than the latencies at the $135^{\circ}$ and $225^{\circ}$ rotations by $t$ test. This is consistent with the hypothesis that the subjects may have switched strategies during some of the trials in Experiment 3, responding during the $180^{\circ}$ rotations by attempting to reverse the target's direction relative to their actual station point.

\section{Conclusions}

People often produce spatially coordinated actions while on the move and plan actions before reaching the station points from which they intend to launch them. While locomoting without vision, observers act as if they perceive the changing self-to-object relations caused by locomotion and have direct access to knowledge of them. Proprioceptive feedback from locomotion facilitates access to knowledge of the spatial structure from novel points, and little or no additional processing is involved. This is the case for the simple rotation movements used in the present experiment and for more complex movements combining rotations and translations in station point (Rieser et al., 1986). For perception while walking without vision, observers have direct access to knowledge of self-to-object relations and, presumably, to knowledge of object-to-object relations, as if they perceive the object-toobject relations of the surrounding environment and their own position is included in the perception.

When observers stay in position and imagine the structure available at novel points of observation, they respond as if they directly access knowledge of the object-to-object relations, which is invariant across the different possible points of observation. For simple translations, little or no additional processing time was needed to judge the directions from the new station. This is as if the subjects scanned their knowledge of the surrounding object-to-object relations and responded by aiming the pointer so that it would parallel the direction of the line connecting the target object and facing direction object. For simple rotations, on the other hand, additional processing was needed, and it varied as a function of the magnitude of rotation between the object's actual station and the to-be-imagined station point. This is as if subjects scanned their knowledge of the surrounding object-to-object relations, identified the facing-object-observer-target-object angle, and rotated that angle (or themselves) to match up with their actual facing direction.
Formally, the representations and computations of analytic geometry provide a way to model access to spatial knowledge under conditions like these. However, the model does not fit the evidence about human performance presented here. For example, the evidence indicates that observers access knowledge of object-to-object relations directly without going through the "origin" of a coordinate system. In addition, the evidence shows that access for imagined rotations is a function of the amount of rotation. The evidence fits better with the idea that analogous processes mediate access to spatial knowledge picked up from perceptual inflow in a situation and knowledge derived from memory about a situation. Many of the similarities have been highlighted for object perception and knowledge (Kosslyn, 1980; Shepard \& Cooper, 1982). More needs to be known about similarities between perception and imagination in the realm of spatial orientation and knowledge of environments explored on foot.

\section{References}

Attneave, F., \& Farrar, P. (1977). The visual world behind the head American Journal of Psychology, 90, 549-563.

Appelle, S. (1972). Perception and discrimination as a function of stimulus orientation: The oblique effect in man and animals. Psychological Bulletin, 78, 266-278.

Brebner, J., Shephard, M., \& Cairney, P. (1972). Spatial relationships and S-R compatibility. Acta Psychologica, 36, 1-15.

Coxeter, H. S. M. (1968). Introduction to geometry. New York: Wiley.

Gibson, J. J. (1979). The ecological approach to visual perception. Boston, MA: Houghton Mifflin.

Hardwick, D. A., McIntyre, C. W., \& Pick, H. L. (1976). Content and manipulation of cognitive maps in children and men. Monographs of the Society for Research in Child Development, 41, 394398.

Hintzman, D., O'Dell, C., \& Arndt, D. (1981). Orientations in cognitive maps. Cognitive Psychology, 13, 149-206.

Howard, I. P., \& Templeton, W. B. (1966). Human spatial orientation. New York: Wiley.

Kosslyn, S. M. (1980). Image and mind. Cambridge, MA: Harvard University Press.

Lee, D. N. (1978). The functions of vision. In H. L. Pick, Jr., \& E. Saltzman (Eds.), Modes of perceiving and processing information (pp. 159-170). New York: Wiley.

Olson, D. R., \& Bialystok, E. (1983). Spatial cognition. Hillsdale, NJ: Erlbaum.

Rieser, J. J., Guth, D. A., \& Hill, E. W. (1986). Sensitivity to perspective structure while walking without vision. Perception, 15, 173-188.

Rieser, J. J. (1987). Mental models and direct perception: Two views of spatial learning without vision. In E. Foulke (Ed.), Proceedings of the Louisville Space Conference (pp. 31-44). Louisville, KY: University of Louisville.

Sedgwick, H. (1983). Environment-centered representation of spatial layout: Available visual information from texture and perspective. In J. Beck, B. Hope, \& A. Rosenfeld (Eds.), Human and machine vision (pp. 425-458). New York: Academic Press.

Shepard, R. N., \& Cooper, L. A. (1982). Mental images and their transformations. Cambridge, MA: MIT Press.

Received August 9, 1988

Revision received February 20, 1989

Accepted March 7, 1989 